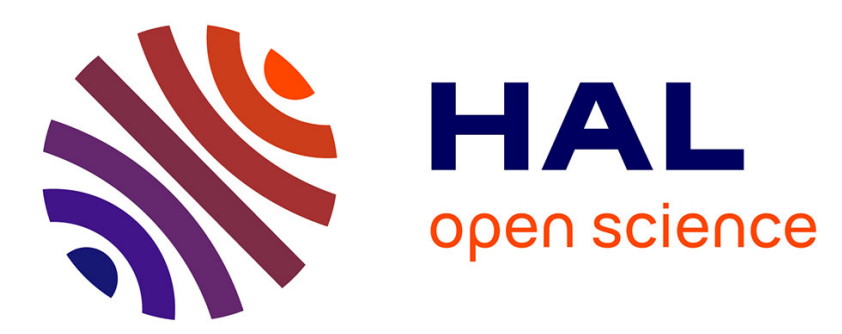

\title{
Channel segregation during columnar solidification: influence of inertia
}

Arvin Kumar, Miha Zaloznik, Hervé Combeau, Benoit Goyeau, Dominique

Gobin

\section{- To cite this version:}

Arvin Kumar, Miha Zaloznik, Hervé Combeau, Benoit Goyeau, Dominique Gobin. Channel segregation during columnar solidification: influence of inertia. 4th International Conference on Porous Media and its Applications in Science and Engineering (ICPM4), Jun 2012, Potsdam, Germany. pp.CD. hal-00845697

\section{HAL Id: hal-00845697 https://hal.science/hal-00845697}

Submitted on 17 Jul 2013

HAL is a multi-disciplinary open access archive for the deposit and dissemination of scientific research documents, whether they are published or not. The documents may come from teaching and research institutions in France or abroad, or from public or private research centers.
L'archive ouverte pluridisciplinaire HAL, est destinée au dépôt et à la diffusion de documents scientifiques de niveau recherche, publiés ou non, émanant des établissements d'enseignement et de recherche français ou étrangers, des laboratoires publics ou privés. 


\title{
Channel Segregation during Columnar Solidification Influence of Inertia
}

\author{
Arvin Kumar*, Miha Založnik*, Hervé Combeau*, B. Goyeau ${ }^{\mathbb{I l}}$ and D. Gobin ${ }^{\mathbb{I}}$ \\ * Institut Jean Lamour - CNRS-Université de Lorraine, 54042 Nancy - France

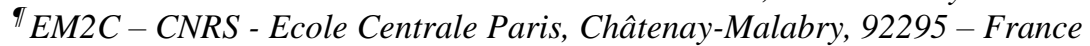

\begin{abstract}
The numerical prediction of channel segregations during two-dimensional columnar solidification of $\mathrm{Sn}-\mathrm{Pb}$ alloy is analyzed when inertia is considered in the dendritic mushy zone. This contribution is included in the momentum transport equation through the quadratic Forchheimer correction term. A significant influence of the Forchheimer term in the vicinity of the liquidus region is found. The natural convective flow field and therefore the global shape of the mushy zone are modified giving rise to significant decrease of channel segregation, due to additional drag in these regions.
\end{abstract}

Keywords: Solidification - Macroscopic model - Forchheimer term - Segregated channels.

PACS: 44.30.+v - 81.30.Fb

\section{INTRODUCTION}

Channel segregates or freckles are considered to be a severe defect in castings because they introduce heterogeneities in the final product properties which cannot be removed in the manufacturing process. In casting solidification, these highly solute concentrated channels appear as long narrow trails oriented in a preferred direction. Their characteristic length scales strongly affect the homogeneity of the casting and therefore the quality and the mechanical properties of the solidifying product can be altered. Channel segregates generally result from instabilities taking place in the interfacial region due to interdendritic liquid flow and by eventual local remelting in the vicinity of the tip of the dendrites [1-9]. The formation of defects in metal casting processes critically depends on the permeability of the mushy zone and on the interdendritic liquid flow. In most solidification models, the columnar dendritic mushy zone is described as a saturated porous medium whose permeability and porosity (liquid volume fraction) are both spatial-dependent. Indeed, the porosity continuously varies from unity in the melt to zero in the solid region leading to variations of permeability ranging over several orders of magnitude.

Macroscopic models of dendritic solidification use the one-domain approach where a single set of conservation equations describes the transport phenomena. In this context, momentum transport is given by the averaged Navier-Stokes equation where the drag in the porous region is taken into account by the Darcy term [3-15, 16]. If the pore Reynolds number $\operatorname{Re}=\rho|\vec{v}| d / \mu$ is larger than unity in some locations of the mushy zone, inertia has to be taken into account through the Forchheimer correction term [17-21].

Although many numerical studies have been performed to investigate channel segregation [1-9, 12], little attention has been given to the influence of inertia on this phenomena. This is the objective of the present study to consider the influence of the Forchheimer correction term on the flow in the mush and on the predictions of channel segregations applied to solidification of $\mathrm{Sn}-\mathrm{Pb}$ alloy in a $2 \mathrm{D}$ rectangular cavity.

\section{MUSH MODELED AS A POROUS MEDIUM}

Let us consider a dendritic columnar solidification case where the dendrites are assumed to be rigid. All the physical properties of the mixture are assumed to be constant and the Boussinesq approximation applies. At the macroscopic scale, the dendritic mushy zone is described as a porous medium of liquid volume fraction $g_{l}$ and permeability $K$. 
The intensity of thermosolutal natural convection in the mush is such that the pore Reynolds number can be greater than unity, and we take account of inertia in the model through the use of the Forchheimer correction term in the momentum transport equation. Using the one-domain modeling approach, this momentum equation for the superficial velocity in the liquid phase takes the form [16]:

$$
\begin{aligned}
& \frac{\rho_{0}}{g_{l}} \frac{\partial \vec{v}}{\partial t}+\frac{1}{g_{l}} \vec{v} \cdot\left(\nabla\left(\frac{\vec{v}}{g_{l}}\right)\right)=-\nabla p+\mu_{e f f} \nabla^{2} \vec{v} \\
& -\left(\frac{\mu}{K}+\frac{\rho_{l} C_{E}}{\sqrt{K}}|\vec{v}|\right) \vec{v} \\
& -\rho_{0}\left[\beta_{T}\left(T-T_{0}\right)+\beta_{C}\left(C_{l}-C_{0}\right)\right] \vec{g}
\end{aligned}
$$

In equation (1), the third and the fourth terms of the right hand side (RHS) represent the Darcy and Forchheimer terms, respectively. $C_{\mathrm{E}}$ in the Forchheimer term is the Ergun constant [22] whose value will be discussed later. In the present study, the permeability is defined using the classical BlakeKozeny relationship:

$$
K=\frac{d^{2} g_{l}^{3}}{180\left(-g_{l}\right)^{2}}
$$

In the dendritic columnar mushy zone, $\mathrm{d}$ in expression (2) corresponds to the interdendritic characteristic arm spacing. Obviously, this expression is very schematic and a more realistic description, accounting for the microstructure of the dendrites, would be necessary. However, this challenging and very important point is out of the scope of the present study.

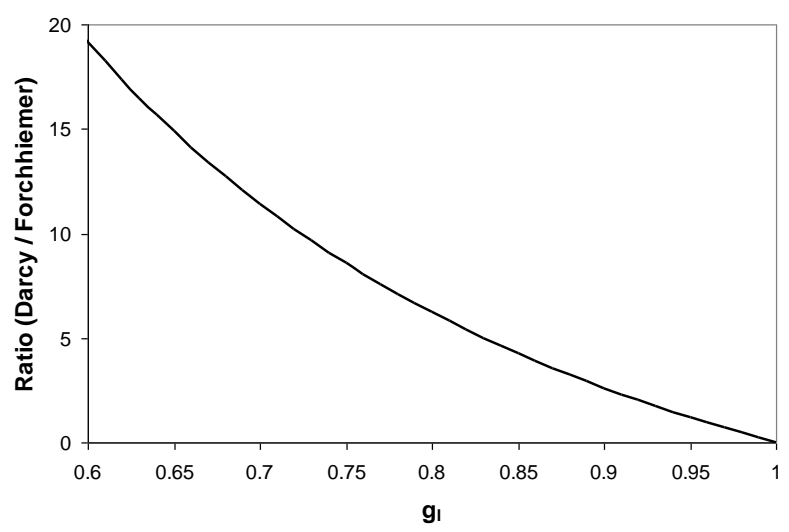

FIGURE 1. Ratio of Darcy and Forchheimer term as a function of liquid fraction

Since our objective is to quantify the influence of inertia on channel segregation, it is relevant to estimate the ratio of the Darcy and the Forchheimer terms:

$$
R=\frac{\text { Darcy }}{\text { Forch. }}=\frac{\sqrt{180}}{C_{E}} \frac{\left(1-g_{l}\right)}{g_{l}^{3 / 2}} \frac{1}{\mathrm{Re}}
$$

This ratio is plotted for $\mathrm{Re}=1$ in Fig. 1 which shows that the Darcy term dominates the Forchheimer term (with $C_{\mathrm{E}}=0.55$ [22]) inside the mushy zone. However, in the vicinity of the liquidus region where the liquid volume fraction is in the range [0.96-1], we may note that the Forchheimer term may reach much larger values than the Darcy term. It can be also noticed that the ratio $\mathrm{R}$ tends to zero as the liquid fraction tends to unity. This implies that, near the liquidus region, the Forchheimer term significantly contributes to the drag and may have an important influence on the channel formation. In the next section, numerical simulations illustrate the contribution of this inertia correction term. For conciseness, the whole solidification model is not presented here and all the details are provided in the following references $[3,9,28]$.

\section{SOLIDIFICATION PROBLEM}

Numerical simulations with and without inertia are performed for solidification of a Sn-5\%wt $\mathrm{Pb}$ binary alloy in a $2 \mathrm{D}$ rectangular $100 \times 60 \mathrm{~mm}^{2}$ cavity (Figure 2).

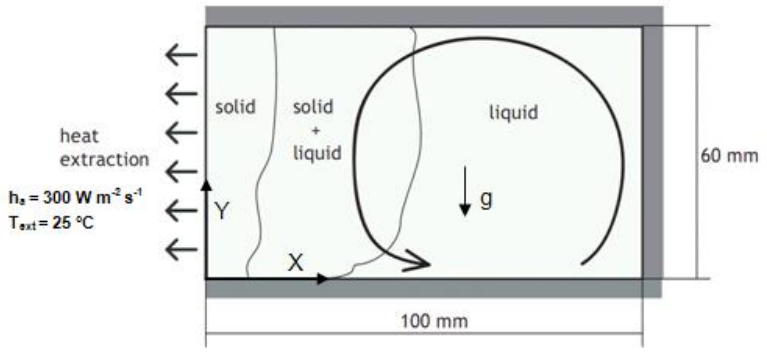

FIGURE 2. Schematic representation of the computational domain for solidification in a $2 \mathrm{D}$ rectangular cavity

This configuration corresponds to the benchmark problem defined by Hebditch and Hunt [23] where channel segregations were experimentally and numerically observed [3, 6, 9]. The cavity is cooled from the left side and the remaining three sides are thermally insulated. The initial temperature $\mathrm{T}_{0}=$ $226^{\circ} \mathrm{C}$ corresponds to an initial superheat of $0.43^{\circ} \mathrm{C}$. For the considered alloy $(\mathrm{Sn}-5 \% \mathrm{wt} \mathrm{Pb})$ the thermal and solutal buoyancy forces are cooperating as the heavier solute $(\mathrm{Pb})$ is rejected into the liquid upon solidification. This thermosolutal configuration tends to create a counter-clockwise flow as schematically shown in Figure 2. The macroscopic model and the numerical method used in the computations are described elsewhere [3, 9]. The thermophysical property data, boundary conditions and parameters 
used in the computations are provided in Table $1[3$, 9]. The influence of the grid size on the solution of the present benchmark (without inertia) has been recently discussed by Kumar et al. [9]. It was found that a fully grid independent solution for prediction of macro- and meso-segregations is very difficult to achieve. However, the global macrosegregation pattern is correctly captured using a $90 \times 90$ mesh. In the present study a $150 \times 150$ mesh is used considering that it constitutes a good compromise between the accuracy of channels prediction and the computational effort. The influence of the grid size on channel segregates in the presence of the Forchheimer term will be discussed at the end of the next section.

\section{RESULTS AND DISCUSSION}

Figure 3 presents the simulated segregation map at the end of solidification for different values of the Ergun constant $(0,0.2,0.55,1.0), C_{\mathrm{E}}=0$ being the case without inertia. It is worth mentioning that $C_{\mathrm{E}}$, which depends both on the microstructure of the porous region and on the local dispersion, is not known a priori and the common value $C_{\mathrm{E}}=0.55$ is generally used [22]. However, the rigorous determination of the Forchheimer effective coefficient can be achieved by solving the closure problem associated to the up-scaling of the local Navier-Stokes equations on a given porous structure [16, 21].

The results presented in Figure 3 are plotted at $t=$ $400 \mathrm{~s}$ when the structure of the channel segregates becomes quasi-stationary [9]. It is shown that the weight of the Forchheimer term (through the Ergun's constant) significantly influences the number of inclined channels. Indeed, if the channels presenting at this time an increase of their average lead composition higher than $1 \% \mathrm{wt}$ are considered, a decrease from 7 to 2 channels is observed when the Ergun constant varies from 0 to 1 . It has been already observed [9] that the channels start to develop at high liquid fraction. Analyzing in terms of the ratio R, we can see that this reduction is mainly due to the strong impact of the Forchheimer contribution in the liquidus region. On the other side, it is interesting to observe that the Forchheimer term hardly affects the overall macrosegregation $\Delta \mathrm{C}$ which is independent of the $C_{\mathrm{E}}$ value. It is estimated that the location of $\mathrm{C}_{\max }$ and $\mathrm{C}_{\min }$ in the cavity is not in the region of inclined channels. The maximum mixture concentration occurs in the left bottom region of the cavity whereas the minimum mixture concentration occurs in the left top region of the cavity.

The nature of segregations with respect to the direction of the double diffusive flow is compared for $C_{\mathrm{E}}=0$ and $C_{\mathrm{E}}=0.55$ in Figure 4 where the streamlines and the segregation map are superimposed. As expected, in both cases, a counter-clockwise convective circulation is observed and the flow penetrates the channels from above. The rejected solute is transported by the flow and the segregation maps reveal a positive macrosegregation in the area below the diagonal from the lower left corner and a negative one above this diagonal. The influence of the

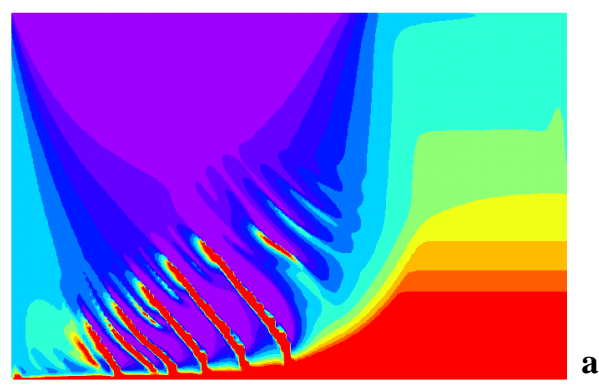

a)
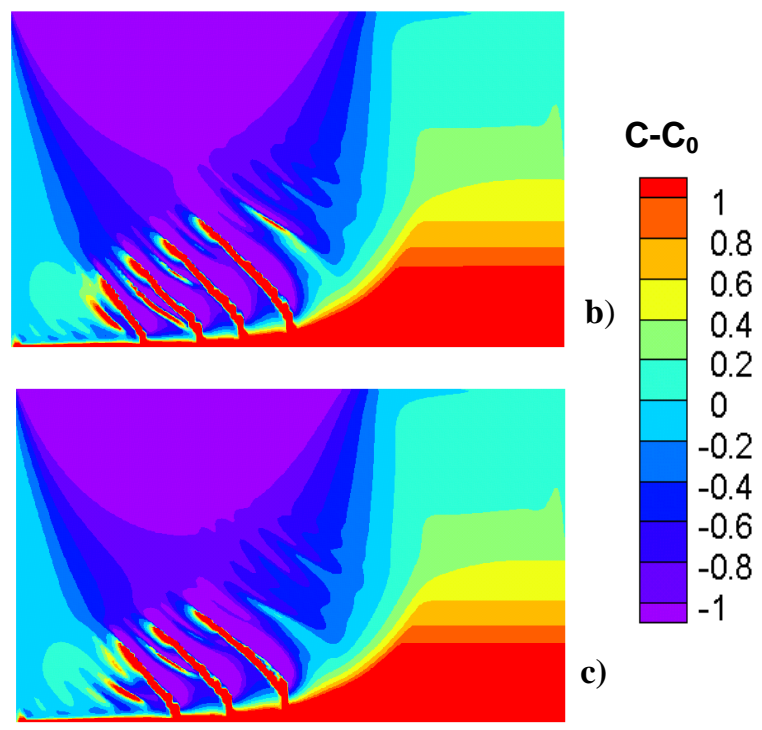

c)

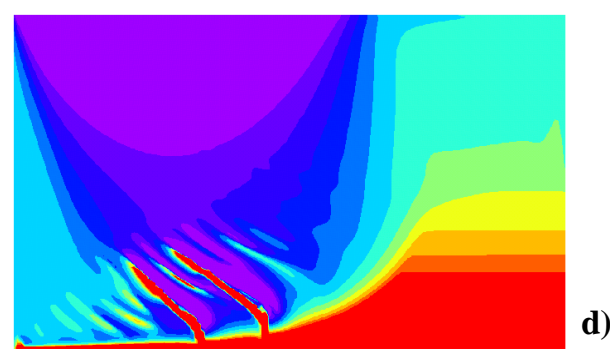

FIGURE 3. Segregation map at the end of solidification (400 s) for simulations with different Ergun constants. (a) $C_{\mathrm{E}}=0$, (b) $C_{\mathrm{E}}=0.2$, (c) $C_{\mathrm{E}}=0.55$, (d) $C_{\mathrm{E}}=1.0$.

The scale shows the $\mathrm{Pb}\left(\mathrm{C}-\mathrm{C}_{0}\right)$ in wt $\%$

flow direction on the segregation was previously studied in the case without inertia $[1,6,7,24]$. In the present case, macrosegregation is characterized by 
liquid velocities oriented in the direction of the temperature gradient such that $\vec{v}_{l} \cdot \nabla T$ be positive (note that the liquidus slope $m$ is negative for the considered alloy). A horizontal channel is found along the bottom wall of the cavity while the inclined channels with a positive mixture concentration form take place in the middle of the cavity. The segregation pattern in the middle of the cavity is of the banded type, alternating positive and negative segregation zones. These banded patterns in the solute field are known as mesosegregations, a severe form of segregation on an intermediate scale.
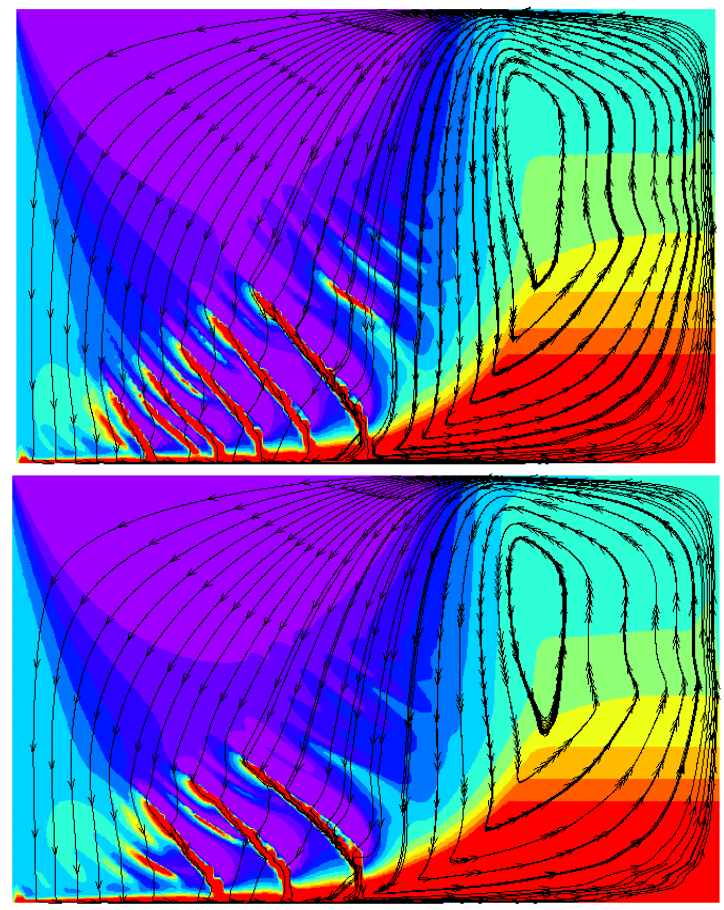

FIGURE 4. Streamlines superimposed on the segregation map at $t=400 \mathrm{~s}$ in the cavity. Top: $\mathrm{C}_{\mathrm{E}}=0$; Bottom: $\mathrm{C}_{\mathrm{E}}=0.55$. Same scale as in Fig. 3.

Figure 5 displays the influence of the Forchheimer term on the concentration profile in the horizontal plane at height $\mathrm{y}=8 \mathrm{~mm}$ where channel segregation takes place. The results show five undulations when inertia is neglected, while only three undulations are present when the Forchheimer contribution is considered. Macrosegregation in the right half of the cavity is similar for both simulations. A map of the drag forces ratio $\mathrm{R}$ at the beginning of channel formation (not shown) confirms that the influence of the Forchheimer correction is found to be larger than the Darcy contribution close to the liquidus where large liquid volume fractions take place, while the Darcy term significantly dominates inside the dendritic mushy layer.

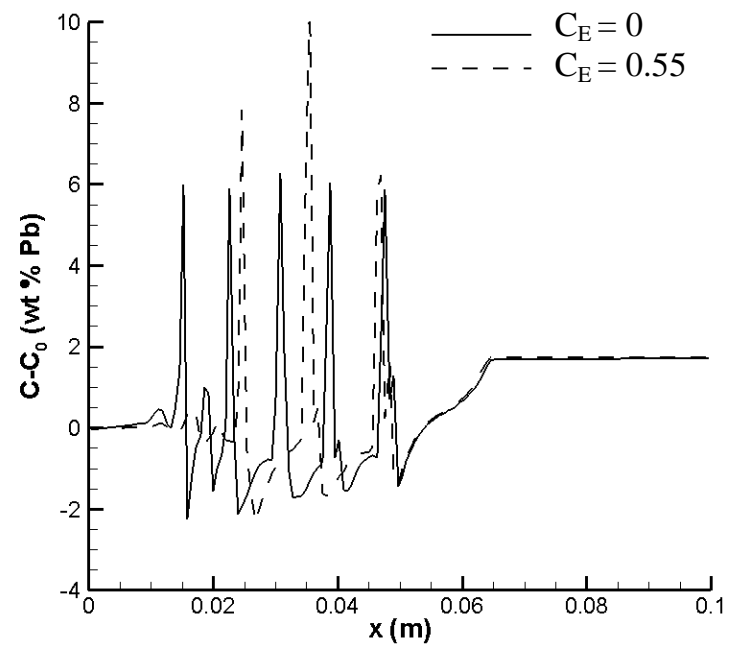

FIGURE 5. Variation of $\mathrm{Pb}$ concentration $\left(\mathrm{C}-\mathrm{C}_{0}\right)$ at $400 \mathrm{~s}$ along a horizontal section at height $\mathrm{y}=8 \mathrm{~mm}$.

Figure 6 illustrates the influence of the Forchheimer term on the flow field at $\mathrm{t}=80 \mathrm{~s}$. The maximum velocity value in the cavity is found to be smaller when inertia is accounted for. Moreover, the flow field is affected even in the fully liquid region. The drag in the porous region accounted for by the Darcy term assumes that the pore Reynolds number $\operatorname{Re}=\rho|\vec{v}| d / \mu$ is lower than unity [3-16].
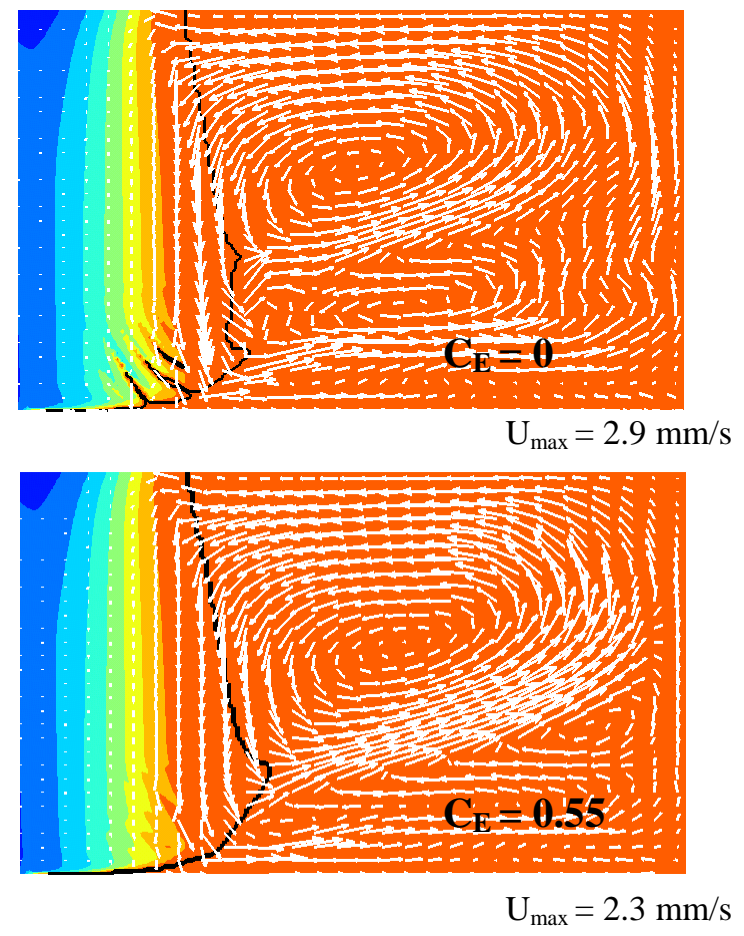

FIGURE 6. Flow field (velocity vectors) in the cavity at $t$ $=80 \mathrm{~s}$. with (bottom) and without (top) the Forchheimer term. The scale shows liquid fraction with the line contour of $g_{1}=1.0$ superimposed. 
In Fig.7, a map of pore Reynolds numbers is shown for the case without inertia which illustrates that in the vicinity of the bulk and close to the mush interface, the pore Reynolds number is larger than unity suggesting that the inertia correction term has to be taken into account. This is why we observe a clear influence of the Forchheimer term on the flow field displayed in Figure 6.

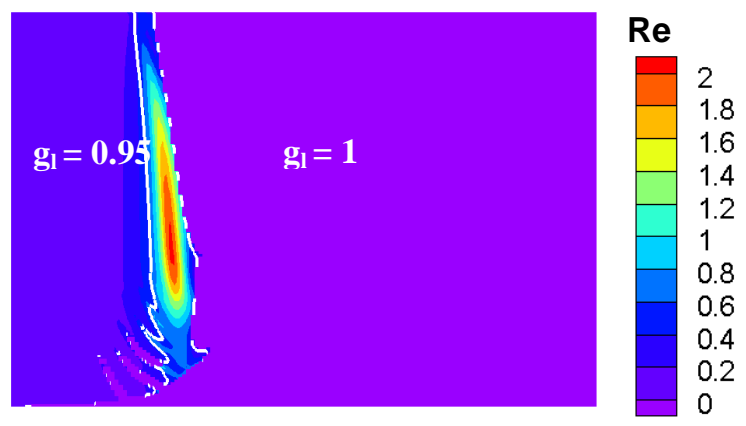

FIGURE 7. Map of pore Reynolds number in the mushy zone at $80 \mathrm{~s}$ for a simulation without the Forchheimer term (maximum pore Reynolds number $=2$ ).

The results presented in this paper were obtained with a $150 \times 150$ mesh. From a general point of view, it is well known that a finer mesh leads to a better spatial approximation particularly in situations where strong gradients are present such as in the dendritic mushy zone where channels form. Calculations performed using two coarser grids $(60 \times 60$ and $90 \times 90$ meshes $)$ clearly show that the number of channels is strongly sensitive to the grid size.

This is probably because we are reaching the limits of the macroscopic model and that the size of the REV is not small enough compared to the spatial variations of the concentrations and liquid fractions in the vicinity of the channels. An alternative to mesh refinement for the description of these channels would consist in developing a downscaling procedure to obtain a zoom of the channel region [25]. Indeed, this method allows the determination of the microscopic fields (solution of the local conservation equations) from the solution of the homogenized model (Darcy scale).

Unlike the channels, the net macrosegregation is found to be insensitive to the inertia contribution, the $\mathrm{C}_{\max }$ and $\mathrm{C}_{\min }$ values being similar whatever the presence of the Forchheimer term. We already noticed that the locations of $\mathrm{C}_{\max }$ and $\mathrm{C}_{\min }$ in the cavity are outside the region of inclined channels where the influence of Forchheimer term is significant. The lack of sensitivity of the net macrosegregation in the whole domain to the inertia contribution may be illustrated by estimated using the global macrosegregation index, GM, given by [9]:

$$
\mathrm{GM}=\frac{1}{\mathrm{C}_{0}}\left[\frac{1}{\mathrm{~V}_{\text {domain }}} \quad \iiint_{\mathrm{V}_{\text {domain }}}\left(\mathrm{C}-\mathrm{C}_{0}\right)^{2} \mathrm{dV}\right]^{1 / 2}
$$

The estimated values of GM for simulations without inertia with $60^{2}, 90^{2}, 150^{2}$ meshes are respectively $33 \%, 34 \%$ and $35.7 \%$ while the corresponding values for simulations with inertia are, respectively, $32.9 \%, 33.8 \%$ and $35.5 \%$. Therefore, we can conclude that although the Forchheimer term significantly influences the predictions of channel segregations, it has a minor influence on the net macrosegregation in the casting.

TABLE 1. Thermophysical data and parameters.

\begin{tabular}{|c|c|}
\hline Parameter & $\mathrm{Sn}-5 \% \mathrm{~Pb}$ \\
\hline \multicolumn{2}{|l|}{ Phase diagram } \\
\hline Initial mass fraction $\left(C_{0}\right), \mathrm{wt} \% \mathrm{~Pb}$ & 5.0 \\
\hline Melting temperature, ${ }^{\circ} \mathrm{C}$ & 232.0 \\
\hline Eutectic temperature, ${ }^{\circ} \mathrm{C}$ & 183.0 \\
\hline Liquidus slope $(m),{ }^{\circ} \mathrm{C}$ wt $\%^{-1}$ & -1.286 \\
\hline Eutectic mass fraction, wt $\% \mathrm{~Pb}$ & 38.1 \\
\hline \multicolumn{2}{|l|}{ Thermophysical data } \\
\hline Specific heat, $\mathrm{J} \mathrm{kg}^{-1} \mathrm{~K}^{-1}$ & 260.0 \\
\hline Thermal conductivity, $\mathrm{W} \mathrm{m}^{-1} \mathrm{~K}^{-1}$ & 55.0 \\
\hline Latent heat of fusion, $\mathrm{J} \mathrm{kg}^{-1}$ & 61000 \\
\hline Reference mass density $(\rho), \mathrm{kg} \mathrm{m}^{-3}$ & 7000.0 \\
\hline $\begin{array}{l}\text { Reference temperature for mass density }\left(T_{0}\right) \text {, } \\
{ }^{\circ} \mathrm{C}\end{array}$ & 226.0 \\
\hline Thermal expansion coefficient $\left(\beta_{\mathrm{T}}\right),{ }^{\circ} \mathrm{C}^{-1}$ & $6.0 \times 10^{-5}$ \\
\hline Solutal expansion coefficient $\left(\beta_{\mathrm{C}}\right)$, wt $\%^{-1}$ & $5.3 \times 10^{-3}$ \\
\hline Dynamic viscosity $(\mu), \mathrm{kg} \mathrm{m}^{-1} \mathrm{~s}^{-1}$ & $1.0 \times 10^{-3}$ \\
\hline $\begin{array}{l}\text { Representative size in the dendritic structure } \\
(d), \mu \mathrm{m}\end{array}$ & 100.0 \\
\hline \multicolumn{2}{|l|}{ Computational parameters } \\
\hline Initial temperature $\left(T_{0}\right),{ }^{\circ} \mathrm{C}$ & 226.0 \\
\hline Heat transfer coefficient $\left(h_{\mathrm{a}}\right), \mathrm{W} \mathrm{m}^{-2} \mathrm{~s}^{-1}$ & 300.0 \\
\hline External temperature $\left(T_{\text {ext }}\right),{ }^{\circ} \mathrm{C}$ & 25.0 \\
\hline Dimension of the cavity $(\mathrm{X} \times \mathrm{Y}), \mathrm{m}$ & $0.1 \times 0.06$ \\
\hline Number of nodes, $\mathrm{X} \times \mathrm{Y}$ directions & $150 \times 150$ \\
\hline
\end{tabular}

These results demonstrate that channel formation is related to the drag in the near-liquidus region where the Forchheimer term is found to be larger than the Darcy term. Since both contributions depend on the local permeability of the dendritic mushy zone, the quality of the description of this latter, especially for large liquid volume fraction, is essential for an accurate prediction of the channels. This point is very challenging since this is typically the region where the experimental estimations of the permeability fail [8, 10, 12]. This illustrates the need for further 
investigation on the representation at the different scales of the dendritic region. Important effort is developed in this direction using both numerical (closure problems [13], phase field [26]) and experimental methods (X-ray imaging [27]). In parallel, benchmark on alloy solidification such as the one presented in [28] are expected to provide important information concerning numerical approximation and comparison with experiments.

\section{CONCLUSION}

This study is devoted to the influence of inertia on the macroscopic representation of columnar dendritic solidification of $\mathrm{Sn}-\mathrm{Pb}$ alloy, more specifically on the prediction of channel formation that takes place in the liquidus region of the mushy zone. As usual, the dendritic mushy zone is modeled as a porous medium and inertia at the macroscopic scale is represented by the quadratic Forchheimer drag term in the momentum equation. Due to the lack of information concerning this drag term, it is described using the Ergun's relationship where the Forchheimer coefficient $C_{\mathrm{F}}=$ $C_{\mathrm{E}}=0.55$. From a general viewpoint, the results show that the Forchheimer term significantly influences the predictions of channel segregations.

More specifically, is has been first observed that the Forchheimer drag is found to be larger than the Darcy drag in the near liquidus regions. Secondly, the number of channel is reduced, from 5 to 3 when inertia is accounted for. Third, the shape of the mushy zone and the magnitude of velocity in the near liquidus and in the fully liquid regions are also affected by the Forchheimer term. Finally, the macrosegregation is hardly influenced by the inertia contribution.

\section{ACKNOWLEDGMENTS}

The authors gratefully acknowledge the financial support of the French Research Agency (ANR) in the frame of Project SMACS (ANR-07-BLAN-190).

\section{REFERENCES}

1. R. Mehrabian, M. Keane, M.C. Flemings, Met.. Trans. 1, 1209-1220 (1970).

2. H. Fredriksson, S.O. Nilsson, Met. Trans. B 9, 111120. (1978)

3. N. Ahmad, H. Combeau, J.-L. Desbiolles, T. Jalanti, G. Lesoult, J. Rappaz, M. Rappaz, C. Stomp, Met. Mat. Trans. A, 29, 617-630 (1998).

4. J. Jain, A. Kumar, P. Dutta, J. Heat Transfer 129, 548558 (2007).

5. J. Jain, A. Kumar, P. Dutta, J. Phys. D: Applied Physics 40, 1150-1160 (2007).
6. M. Založnik, H. Combeau, "Effects of solidification kinetics and liquid density in modeling of macrosegregation in castings", in MC-WASP XII-TMS (Warrendale-PA, USA, 2009), 253-260.

7. M. Založnik, H. Combeau, Int. J. Thermal Sci. 49, 1500-1509 (2010).

8. R.F. Katz, M.G. Worster, J. Comp. Phys. 227, 98239840 (2008).

9. A. Kumar, B. Dussoubs, M. Založnik, H. Combeau, J. Phys. D: Appl. Phys. 42, 105503 (2009).

10. D.R Poirier, Met. Trans. B 18, 245-256 (1987).

11. C. Prakash, V.R. Voller, Num. Heat Transfer 15, 171189 (1989).

12. M.C. Schneider, C. Beckermann, Int. J. Heat Mass Transfer, 38 3455-3473 (1995).

13. B. Goyeau, T. Benihaddadene, D. Gobin, M. Quintard, Met. Mat. Trans. B 30, 613-622 (1999).

14. S. Chakraborty, P. Dutta, Int. J. Heat Mass Transfer 46, 2115-2134 (2003).

15. A. Kumar, P. Dutta, J. Phys. D: Appl. Phys. 41, 155501 (2008).

16. B. Goyeau, P. Bousquet-Melou, M. Quintard, D. Gobin, F. Fichot, Computational and Applied Mathematics 23, 381-400 (2004).

17. C. Beckermann, R. Viskanta, Int. J. Heat Mass Transfer, 31, 35-46 (1988).

18. D.A. Nield, Transport in Porous Media 43, 597-601 (2001)

19. P. Bousquet-Melou, B. Goyeau, M. Quintard, F. Fichot, D. Gobin, Int. J. Heat Mass Transfer 45, 3651-3665 (2002).

20. P. Forchheimer P. Wasserbewegung durch boden, $Z$. Ver. Deutsch. Ing. 45, 1782-1788 (1901).

21. S. Whitaker, Transport in Porous Media 25, 27-61 (1996).

22. S. Ergun, Chem. Process Eng. 48, 89-94 (1952).

23. D. Hebditch, J. Hunt, Met. Trans. 5, 1557-1564 (1974).

24. H. Combeau, M. Založnik, S. Hans, P.E. Richy, , Met. Mat. Trans. B 40, 289-304 (2009).

25. P.E. Angeli, O. Cioni, F. Ducros, B. Goyeau, "Downscaling method from macroscopic to microscopic scale in a periodic two-dimensional porous medium" in 3rd ECI International Conference on Porous Media and its Applications to Science, Engineering and Industry (Montecatini, Italy, June 20$25,2010)$.

26. M.D. Asta, C. Beckermann, A. Karma, W. Kurz, R. Napolitano, M. Plapp, G. Purdy, M. Rappaz, R. Trivedi,, Acta Materialia 57, 941-971 (2009).

27. G. Quillet, A. Ciobanas, P. Lehmann, Y. Fautrelle, Int. J. Heat Mass Transfer 50, 654-666 (2007).

28. M. Bellet, H. Combeau, Y. Fautrelle, D. Gobin, M. Rady, E. Arquis, O. Budenkova, B. Dussoubs, Y. Duterrail, A. Kumar, C.A. Gandin, B. Goyeau, S. Mosbah, M. Založnik, Int. J. Thermal Sci. 48, 20132016 (2009). 